\title{
REGULARITY OF NONLINEAR EQUATIONS FOR FRACTIONAL LAPLACIAN
}

\author{
ALIANG XIA AND JIANFU YANG \\ (Communicated by James E. Colliander)
}

Abstract. In this paper, we prove that any $H^{s}(\Omega)$ solution $u$ of the problem

$$
(-\Delta)^{s} u=f(u) \text { in } \Omega, \quad u=0 \text { on } \partial \Omega,
$$

belongs to $L^{\infty}(\Omega)$ for the nonlinearity of $f(t)$ being subcritical and critical. This implies that the solution $u$ is classical if $f(t)$ is $C^{1, \gamma}$ for some $0<\gamma<1$.

\section{INTRODUCTION}

In this paper, we consider the regularity of solutions to the problem involving fractional Laplacian operators

$$
\begin{cases}(-\Delta)^{s} u=f(u), & x \in \Omega, \\ u=0, & x \in \partial \Omega,\end{cases}
$$

where $\Omega \subset \mathbb{R}^{N}, N \geq 2$, is a smooth bounded domain, $0<s<1$. The fractional operator $(-\Delta)^{s}$ is defined as follows. Let $\varphi_{k}$ be an eigenfunction of $-\Delta$ given by

$$
\begin{cases}-\Delta \varphi_{k}=\mu_{k} \varphi_{k}, & x \in \Omega, \\ \varphi_{k}=0, & x \in \partial \Omega,\end{cases}
$$

where $\mu_{k}$ is the corresponding eigenvalue of $\varphi_{k}, 0<\mu_{1}<\mu_{2} \leq \mu_{3} \leq \cdots \leq \mu_{k} \rightarrow$ $+\infty$. Then, $\left\{\varphi_{k}\right\}_{k=1}^{\infty}$ is an orthonormal basis of $L^{2}(\Omega)$ satisfying $\int_{\Omega} \varphi_{j} \varphi_{k} d x=\delta_{j, k}$. We define the operator $(-\Delta)^{s}$ for any $u \in C_{c}^{\infty}(\Omega)$ by

$$
(-\Delta)^{s} u=\sum_{k=1}^{\infty} \mu_{k}^{s} u_{k} \varphi_{k}
$$

where

$$
u=\sum_{k=1}^{\infty} u_{k} \varphi_{k} \text { and } \quad u_{k}=\int_{\Omega} u \varphi_{k} d x .
$$

This operator can be extended by density for $u$ in the Hilbert space

$$
H=\left\{u \in L^{2}(\Omega):\|u\|_{H}^{2}=\sum_{k=1}^{\infty} \mu_{k}^{s}\left|u_{k}\right|^{2}<+\infty\right\} .
$$

Received by the editors February 6, 2011.

2010 Mathematics Subject Classification. Primary 35J25, 47G30, 35B45, 35J70.

Key words and phrases. Regularity, $L^{\infty}$ bounds, fractional Laplacian.

The second author is supported by National Natural Sciences Foundations of China, grant No. 11271170, and the GAN PO 555 program of Jiangxi. 
It is known that

$$
H= \begin{cases}H^{s}(\Omega), \text { if } & s \in\left(0, \frac{1}{2}\right), \\ H_{00}^{\frac{1}{2}}(\Omega), \text { if } & s=\frac{1}{2}, \\ H_{0}^{s}(\Omega), \text { if } & s \in\left(\frac{1}{2}, 1\right) .\end{cases}
$$

The fractions of the Laplacian are the infinitesimal generators of Lévy stable diffusion processes and appear in anomalous diffusions in plasmas, flames propagation and chemical reactions in liquid, and American options in finances.

An important feature of the operator $(-\Delta)^{s}$ is its nonlocal character, which can be realized as the boundary operator of a suitable extension in the half-cylinder $\Omega \times$ $(0, \infty)$. Such an interpretation was demonstrated in 5 for the fractional Laplacian in $\mathbb{R}^{N}$. Their construction can be extended to the case of bounded domains as in 6]. Indeed, let us define

$$
\mathcal{C}=\Omega \times(0,+\infty), \quad \partial_{L} \mathcal{C}=\partial \Omega \times[0,+\infty) .
$$

We write points in the cylinder $\mathcal{C}$ by $(x, y) \in \mathcal{C}=\Omega \times(0,+\infty)$. Given $s \in(0,1)$, consider the space $H_{0, L}^{1}\left(y^{1-2 s}\right)$ of measurable functions $v: \mathcal{C} \rightarrow \mathbb{R}$ such that $v \in$ $H^{1}(\Omega \times(s, t))$ for all $0<s<t<+\infty, v=0$ on $\partial_{L} \mathcal{C}$ and for which the following norm is finite:

$$
\|v\|_{H_{0, L}^{1}\left(y^{1-2 s}\right)}^{2}=\int_{\mathcal{C}} y^{1-2 s}|\nabla v|^{2} d x d y .
$$

Hence, we can study problem (1.1) by variational methods for a local problem. More precisely, problem (1.1) can be reduced to the problem

$$
\begin{cases}\operatorname{div}\left(y^{1-2 s} \nabla v\right)=0, & \text { in } \quad \mathcal{C}, \\ v=0, & \text { on } \partial_{L} \mathcal{C}, \\ y^{1-2 s} \frac{\partial v}{\partial \nu}=f(u), & \text { in } \Omega \times\{0\},\end{cases}
$$

where $\nu$ is the unit outer normal to $\Omega \times\{0\}$. If $v$ satisfies (1.4), then the trace $v$ on $\Omega \times\{0\}$ of the function $v$ will be a solution of problem (1.4).

Recently, problem (1.1) has been studied in [2], 3, 44, 5] and [6], etc., where the existence of solutions and various properties of solutions were considered. In particular, the regularity of weak solutions of (1.1) was considered in [3] and [6]. It proved in [3] and [6] the following results.

Theorem 1.1. Let $f=h \in H^{\prime}$ and $u \in H$ be a solution of (1.1).

(i) If $h \in L^{\infty}(\Omega)$, then $u \in C^{\alpha}(\bar{\Omega})$, where $0<\alpha<1$.

(ii) If $h \in C^{\alpha}(\Omega)$, then $u \in C^{2, \alpha}(\Omega) \cap C^{\alpha}(\bar{\Omega})$ for some $0<\alpha<1$.

According to Theorem 1.1 if a weak solution $u$ of (1.1) belongs to $L^{\infty}(\Omega), u$ will be Hölder continuous provided that $f$ is continuous. We may also verify from the proof of Proposition 2.9 in [9] that $u \in C_{l o c}^{\alpha}(\Omega)$ if $u \in L_{l o c}^{\infty}(\Omega)$ and so on. In [4], it was shown for the half Laplacian, i.e. $s=\frac{1}{2}$, and the subcritical case that solutions are bounded in $L^{q}$ norm for all $q<\infty$ by the Brézis-Kato argument [1]. It seems that the $L^{q}$ bound in 4 cannot be simply improved to be a $L^{\infty}$ bound by their argument. For the general case $s \neq \frac{1}{2}$, in [6] the authors suppose, among other things, that $f(t)$ is nondecreasing and that

$$
\lim _{t \rightarrow+\infty} \frac{f(t)}{t}=+\infty
$$


They showed the extremal solution of (1.1) is bounded for the lower dimensional case, and for the higher dimensional case, the solution is possibly singular; see Theorem 1.4 in [6]. Actually, if $f(t)=e^{t}, n=10$, the extremal solution is singular if $\Omega=B_{1}(0)$. We will prove in this paper that any weak solution of (1.1) is classical in subcritical and critical cases for the dimension $n \geq 2$. By a weak solution of (1.1) we mean a solution belonging to $H^{s}(\Omega)$, or alternatively, an $H_{0, L}^{1}\left(y^{1-2 s}\right)$ solution of (1.5). We suppose in this paper that $f \in C^{1, \gamma}(\mathbb{R}), \gamma>\max \{0,1-2 s\}$, satisfies

$$
|f(t)| \leq C\left(1+|t|^{p}\right)
$$

where $1<p \leq \frac{N+2 s}{N-2 s}$. By the Pohozaev identity, we know that $2_{s}^{*}=\frac{2 N}{n-2 s}$ is the critical exponent for $(-\Delta)^{s}$. The restriction of the exponent $p$ is natural in the sense of variational methods. Our main result is the following.

Theorem 1.2. Suppose condition (1.5) holds. Let $u \in H^{s}(\Omega)$ be a solution of (1.1). Then, $u \in L^{\infty}(\Omega)$ if $1<p<\frac{N+2 s}{N-2 s}$; and $u \in L_{l o c}^{\infty}(\Omega)$ if $p=\frac{N+2 s}{N-2 s}$. Consequently, $u \in C_{\text {loc }}^{2, \alpha}(\Omega)$ for some $0<\alpha<1$.

By Theorem 1.2. we see that if the growth of $f(t)$ is not so fast at infinity, solutions of (1.1) are classical without the restriction on the dimension of the whole space $\mathbb{R}^{N}$. In the critical case, it is a Brézis-Kato type result for a fractional Laplacian. Our argument is the use of Moser-Nash iteration for problem (1.4). Although the weighted function $y^{1-2 s}$ is possibly singular or degenerates at $y=0$, we still may establish an inverse Hölder inequality for $v(\cdot, 0)=u(\cdot)$, and we may iterate the inequality for $u$. The proof of Theorem 1.2 is given in section 2 .

\section{Proof of Theorem 1.2}

In this section, we will prove Theorem 1.2, First, we recall a result in [6].

Lemma 2.1. There exists a trace operator from $H_{0, L}^{1}\left(y^{1-2 s}\right)$ into $H_{0}^{s}(\Omega)$. Furthermore, the space $H$ given by (1.2) is characterized by

$$
H=\left\{u=\operatorname{tr}_{\Omega} v: v \in H_{0, L}^{1}\left(y^{1-2 s}\right)\right\} .
$$

Lemma 2.1 was proved in 6]. In its proof, we see in fact that the mapping tr : $H_{0, L}^{1}\left(y^{1-2 s}\right) \hookrightarrow H^{s}(\Omega)$ is continuous. Next, we have the Sobolev embedding theorem.

Lemma 2.2. Given $s>0$ and $\frac{1}{\sigma} \geq 1$ so that $\frac{1}{\sigma} \geq \frac{1}{2}-\frac{s}{N}$, the inclusion map $i: H^{s}(\Omega) \rightarrow L^{\sigma}(\Omega)$ is well defined and bounded. If the above inequality is strict, then the inclusion is compact.

By Lemma 2.1 and Lemma 2.2, we know that there exists a continuous linear mapping from $H_{0, L}^{1}\left(y^{1-2 s}\right)$ to $L^{q}(\Omega)$ if $2 \leq q \leq \frac{2 N}{N-2 s}$.

Proof of Theorem 1.2. We first deal with the subcritical case $1 \leq p<\frac{N+2 s}{N-2 s}$.

Let $\bar{t}=|t|+k$ and $\bar{t}^{+}=t^{+}+k$. For $k>0$ large, we have

$$
|f(t)| \leq C|\bar{t}|^{p},
$$

where $1<p<\frac{N+2 s}{N-2 s}$. Denote $v^{+}=\max \{0, v\}, v^{-}=-\min \{0, v\}$. We deal only with $v^{+}$; it can be done in the same way for $v^{-}$. Let

$$
\bar{v}_{L}^{+}=\left\{\begin{array}{lll}
\bar{v}^{+} & \text {if } & \bar{v}^{+}<L, \\
L & \text { if } & \bar{v}^{+} \geq L .
\end{array}\right.
$$


For any $\varphi \in H_{0, L}^{1}\left(y^{1-2 s}\right)$, by (1.4),

$$
\int_{\mathcal{C}} y^{1-2 s} \nabla v \nabla \varphi d x d y=\int_{\Omega \times\{0\}} y^{1-2 s} \frac{\partial v}{\partial y} \varphi d x=\int_{\Omega \times\{0\}} f(u) \varphi d x .
$$

For $\beta>1$ to be determined, we choose in (2.2) that

$$
\varphi=\bar{v}^{+}\left(\bar{v}_{L}^{+}\right)^{2(\beta-1)}-k^{2(\beta-1)+1},
$$

and since

$$
\nabla \varphi=\left(\bar{v}_{L}^{+}\right)^{2(\beta-1)} \nabla \bar{v}^{+}+2(\beta-1) \bar{v}^{+}\left(\bar{v}_{L}^{+}\right)^{2(\beta-1)-1} \nabla \bar{v}_{L}^{+},
$$

we obtain

$$
\begin{aligned}
& \int_{\mathcal{C}} y^{1-2 s} \nabla v\left(\left(\bar{v}_{L}^{+}\right)^{2(\beta-1)} \nabla \bar{v}^{+}+2(\beta-1) \bar{v}^{+}\left(\bar{v}_{L}^{+}\right)^{2(\beta-1)-1} \nabla \bar{v}_{L}^{+}\right) d x d y \\
& =\int_{\mathcal{C}} y^{1-2 s}\left(\left(\bar{v}_{L}^{+}\right)^{2(\beta-1)}\left|\nabla \bar{v}^{+}\right|^{2}+2(\beta-1) \bar{v}^{+}\left(\bar{v}_{L}^{+}\right)^{2(\beta-1)}\left|\nabla \bar{v}_{L}^{+}\right|^{2}\right) d x d y .
\end{aligned}
$$

Let $w_{L}=\bar{v}^{+}\left(\bar{v}_{L}^{+}\right)^{\beta-1}$ and then

$$
\nabla w_{L}=\left(\bar{v}_{L}^{+}\right)^{\beta-1} \nabla v^{+}+(\beta-1) \bar{v}^{+}\left(\bar{v}_{L}^{+}\right)^{\beta-2} \nabla v_{L}^{+} ;
$$

we deduce from (2.2) and (2.3) for $\beta>1$ that

$$
\begin{aligned}
& \int_{\mathcal{C}} y^{1-2 s}\left|\nabla w_{L}\right|^{2} d x d y \\
& \leq C \beta \int_{\mathcal{C}} y^{1-2 s} \nabla v\left(\left(\bar{v}_{L}^{+}\right)^{2(\beta-1)}\left|\nabla \bar{v}^{+}\right|^{2}+2(\beta-1)\left(\bar{v}_{L}^{+}\right)^{2(\beta-1)}\left|\nabla \bar{v}_{L}^{+}\right|^{2}\right) d x d y \\
& =C \beta \int_{\Omega \times\{0\}}\left(\bar{v}^{+}\left(\bar{v}_{L}^{+}\right)^{2(\beta-1)}-k^{2(\beta-1)+1}\right) f(v) d x d y \\
& \leq C \beta \int_{\Omega \times\{0\}} \bar{v}^{+}\left(\bar{v}_{L}^{+}\right)^{2(\beta-1)}|f(v)| d x d y .
\end{aligned}
$$

By the assumption on $f$, for any $\varepsilon>0$, there exists $C_{\varepsilon}>0$ so that

$$
|f(t)| \leq C|\bar{t}|^{p} \leq \varepsilon|\bar{t}|^{2_{s}^{*}-1}+C_{\varepsilon}|\bar{t}| .
$$

This implies that

$$
\begin{aligned}
& \int_{\Omega \times\{0\}} \bar{v}^{+}\left(\bar{v}_{L}^{+}\right)^{2(\beta-1)}|f(v)| d x \\
& \leq C \int_{\Omega \times\{0\}}\left|\bar{v}^{+}\right|^{p+1}\left(\bar{v}_{L}^{+}\right)^{\beta-1} d x \\
& \leq C \int_{\Omega \times\{0\}}\left[\varepsilon\left(\bar{v}^{+}\right)^{2_{s}^{*}}\left(\bar{v}_{L}^{+}\right)^{2(\beta-1)}+C_{\varepsilon}\left(\bar{v}_{L}^{+}\right)^{2(\beta-1)}\left(\bar{v}^{+}\right)^{2}\right] d x .
\end{aligned}
$$

By (2.4), (2.5) and the Sobolev embedding theorem,

$$
\begin{aligned}
& \left(\int_{\Omega \times\{0\}}\left|w_{L}\right|^{2_{s}^{*}} d x\right)^{\frac{2}{2_{s}^{*}}} \leq C \int_{\mathcal{C}} y^{1-2 s}\left|\nabla w_{L}\right|^{2} d x d y \\
& \leq C \beta \int_{\Omega \times\{0\}}\left[\varepsilon\left(\bar{v}^{+}\right)^{2_{s}^{*}}\left(\bar{v}_{L}^{+}\right)^{2(\beta-1)}+C_{\varepsilon}\left(\bar{v}_{L}^{+}\right)^{2(\beta-1)}\left(\bar{v}^{+}\right)^{2}\right] d x .
\end{aligned}
$$


That is,

$$
\begin{aligned}
& \left(\int_{\Omega}\left(\bar{u}^{+}\left(\bar{u}_{L}^{+}\right)^{\beta-1}\right)^{2_{s}^{*}} d x\right)^{\frac{2}{2_{s}^{*}}} \\
& \leq C \beta \int_{\Omega}\left[\varepsilon\left(\bar{u}^{+}\right)^{2_{s}^{*}}\left(\bar{u}_{L}^{+}\right)^{2(\beta-1)}+C_{\varepsilon}\left(\bar{u}_{L}^{+}\right)^{2(\beta-1)}\left(\bar{u}^{+}\right)^{2}\right] d x .
\end{aligned}
$$

We claim that $\bar{u}^{+} \in L^{\frac{\left(2_{s}^{*}\right)^{2}}{2}}(\Omega)$. Indeed, choosing $\beta=\frac{2_{s}^{*}}{2}$, we have

$$
\begin{aligned}
& \left(\int_{\Omega}\left(\bar{u}^{+}\left(\bar{u}_{L}^{+}\right)^{\frac{2_{s}^{*}-2}{2}}\right)^{2_{s}^{*}} d x\right)^{\frac{2}{2_{s}^{*}}} \\
& \leq \int_{\Omega}\left[\varepsilon\left(\bar{u}^{+}\right)^{2_{s}^{*}}\left(\bar{u}_{L}^{+}\right)^{2_{s}^{*}-2}+C_{\varepsilon}\left(\bar{u}_{L}^{+}\right)^{2_{s}^{*}-2}\left(\bar{u}^{+}\right)^{2}\right] d x \\
& \leq \varepsilon\left(\int_{\Omega}\left(\bar{u}^{+}\left(\bar{u}_{L}^{+}\right)^{\frac{2_{s}^{*}-2}{2}}\right)^{2_{s}^{*}} d x\right)^{\frac{2}{2_{s}^{*}}}\left(\int_{\Omega}\left(\bar{u}^{+}\right)^{2_{s}^{*}} d x\right)^{\frac{2_{s}^{*}-2}{2_{s}^{*}}}+C_{\varepsilon} \int_{\Omega}\left(\bar{u}_{L}^{+}\right)^{2_{s}^{*}-2}\left(\bar{u}^{+}\right)^{2} d x .
\end{aligned}
$$

Choosing $\varepsilon>0$ properly small, we obtain

$$
\left(\int_{\Omega}\left(\bar{u}^{+}\left(\bar{u}_{L}^{+}\right)^{\frac{2_{s}^{*}-2}{2}}\right)^{2_{s}^{*}} d x\right)^{\frac{2}{2_{s}^{*}}} \leq C \int_{\Omega}\left(\bar{u}_{L}^{+}\right)^{2_{s}^{*}-2}\left(\bar{u}^{+}\right)^{2} d x .
$$

Let $L \rightarrow+\infty$. It yields

$$
\left(\int_{\Omega}\left(\bar{u}^{+}\right)^{\frac{\left(\left(2 *_{s}^{*}\right)^{2}\right.}{2}} d x\right)^{\frac{2}{2_{s}^{*}}} \leq C \int_{\Omega}\left(\bar{u}^{+}\right)^{2_{s}^{*}} d x<+\infty .
$$

Now let $t=\frac{\left(2_{s}^{*}\right)^{2}}{2\left(2_{s}^{*}-2\right)}$; it follows that $\frac{2 t}{t-1}<2_{s}^{*}$. We estimate the right-hand side of (2.6). By the Hölder inequality,

$$
\begin{aligned}
& \int_{\Omega}\left(\bar{u}^{+}\right)^{2_{s}^{*}}\left(\bar{u}_{L}^{+}\right)^{2(\beta-1)} d x \\
& \leq\left(\int_{\Omega}\left(\bar{u}^{+}\right)^{\left(2_{s}^{*}-2\right) t} d x\right)^{\frac{1}{t}}\left(\int_{\Omega}\left(\bar{u}^{+}\right)^{\frac{2 \beta t}{t-1}} d x\right)^{1-\frac{1}{t}} \\
& \leq C\left(\int_{\Omega}\left(\bar{u}^{+}\right)^{\frac{2 \beta t}{t-1}} d x\right)^{1-\frac{1}{t}}
\end{aligned}
$$

and

$$
\begin{aligned}
& \int_{\Omega}\left(\bar{u}^{+}\right)^{2 \beta} d x \\
& \leq\left(\int_{\Omega}\left(\bar{u}^{+}\right)^{\left(2_{s}^{*}-2\right) t} d x\right)^{\frac{1}{t}}\left(\int_{\Omega}\left(\bar{u}^{+}\right)^{\frac{2 \beta t}{t-1}} d x\right)^{1-\frac{1}{t}} \\
& \leq C\left(\int_{\Omega}\left(\bar{u}^{+}\right)^{\frac{2 \beta t}{t-1}} d x\right)^{1-\frac{1}{t}} .
\end{aligned}
$$

We deduce from (2.6), (2.9) and (2.10) that

$$
\left(\int_{\Omega}\left(\bar{u}^{+}\left(\bar{u}_{L}^{+}\right)^{\beta-1}\right)^{2_{s}^{*}} d x\right)^{\frac{2}{2_{s}^{*}}} \leq C \beta^{2}\left(\int_{\Omega}\left(\bar{u}^{+}\right)^{\frac{2 \beta t}{t-1}} d x\right)^{1-\frac{1}{t}},
$$

namely,

$$
\left(\int_{\Omega}\left(\bar{u}^{+}\right)^{\beta 2_{s}^{*}} d x\right)^{\frac{1}{\beta}} \leq C^{\frac{1}{\beta}} \beta^{\frac{2_{s}^{*}}{\beta}}\left(\int_{\Omega}\left(\bar{u}^{+}\right)^{\frac{2 \beta t}{t-1}} d x\right)^{\frac{(t-1) 2_{s}^{*}}{2 t \beta}} .
$$


Let $\tau \frac{2-s^{*}(t-1)}{2 t}, \tau>1$, and

$$
I_{i}=\left(\int_{\Omega}\left(\bar{u}^{+}\right)^{\frac{2 \tau^{i} t}{t-1}} d x\right)^{\frac{1}{\tau^{i}}} .
$$

Iterating by (2.11), we obtain

$$
\begin{aligned}
I_{i+1} & \leq C^{\frac{1}{\tau^{i+1}}}\left(\tau^{2_{s}^{*} i}\right)^{\frac{1}{\tau^{i+1}}} I_{i} \\
& \leq C^{\sum_{j=0}^{i+1} \frac{1}{\tau^{j}}} e^{\sum_{j=0}^{i+1} 2_{s}^{*} j \frac{1}{\tau^{j}}} I_{0}
\end{aligned}
$$

and

$$
I_{0}=\int_{\Omega}\left(\bar{u}^{+}\right)^{\frac{2 t}{t-1}} d x<\infty .
$$

Next, we consider the critical case: $p=\frac{N+2 s}{N-2 s}$.

For any $x_{0} \in \Omega$, we choose $R>0$ small so that $B_{2 R}\left(x_{0}\right) \subset \Omega$. Let $\eta \in C_{0}^{\infty}(\Omega)$ be such that $\eta(x) \equiv 1$ if $x \in B_{R}\left(x_{0}\right)$ and $\eta(x) \equiv 0$ if $x \notin B_{R+r}\left(x_{0}\right)$. Furthermore, $|\nabla \eta| \leq \frac{2}{r}$, where $0<r<R$ and $\eta \geq 0$ in $\Omega$. Choose in (2.2) that

$$
\varphi=\eta^{2}\left(\bar{v}^{+}\left(\bar{v}_{L}^{+}\right)^{2(\beta-1)}-k^{2(\beta-1)+1}\right),
$$

and since

$$
\begin{aligned}
\nabla \varphi & =\eta^{2}\left[\left(\bar{v}_{L}^{+}\right)^{2(\beta-1)} \nabla \bar{v}^{+}+2(\beta-1) \bar{v}^{+}\left(\bar{v}_{L}^{+}\right)^{2(\beta-1)-1} \nabla \bar{v}_{L}^{+}\right] \\
& +2 \eta \nabla_{x} \eta\left(\bar{v}^{+}\left(\bar{v}_{L}^{+}\right)^{2(\beta-1)}-k^{2(\beta-1)+1}\right)
\end{aligned}
$$

we obtain

$$
\begin{aligned}
& \int_{\mathcal{C}} y^{1-2 s} \eta^{2}\left(\left(\bar{v}_{L}^{+}\right)^{2(\beta-1)}\left|\nabla \bar{v}^{+}\right|^{2}+2(\beta-1)\left(\bar{v}_{L}^{+}\right)^{2(\beta-1)}\left|\nabla \bar{v}_{L}^{+}\right|^{2}\right) d x d y \\
& +\int_{\mathcal{C}} y^{1-2 s} 2 \eta \nabla v \nabla_{x} \eta\left(\bar{v}^{+}\left(\bar{v}_{L}^{+}\right)^{2(\beta-1)}-k^{2(\beta-1)+1}\right) d x d y \\
& =\int_{\Omega \times\{0\}} \eta^{2}\left(\bar{v}^{+}\left(\bar{v}_{L}^{+}\right)^{2(\beta-1)}-k^{2(\beta-1)+1}\right) f(v) d x d y .
\end{aligned}
$$

Hence, by the assumption on $f,|f(t)| \leq C|\bar{t}|^{2_{s}^{*}-1}$,

$$
\begin{aligned}
& \int_{\mathcal{C}} y^{1-2 s} \eta^{2}\left(\left(\bar{v}_{L}^{+}\right)^{2(\beta-1)}\left|\nabla \bar{v}^{+}\right|^{2}+2(\beta-1)\left(\bar{v}_{L}^{+}\right)^{2(\beta-1)}\left|\nabla \bar{v}_{L}^{+}\right|^{2}\right) d x d y \\
& \leq \int_{\Omega \times\{0\}} \eta^{2}\left(\bar{v}^{+}\right)^{2_{s}^{*}}\left(\bar{v}_{L}^{+}\right)^{2(\beta-1)} d x+\int_{\mathcal{C}} y^{1-2 s}\left|\nabla_{x} \eta\right|^{2}\left(\bar{v}^{+}\right)^{2}\left(\bar{v}_{L}^{+}\right)^{2(\beta-1)} d x d y .
\end{aligned}
$$

Let $w_{L}=\eta \bar{v}^{+}\left(\bar{v}_{L}^{+}\right)^{\beta-1}$. We infer that

$$
\begin{aligned}
& \int_{\mathcal{C}} y^{1-2 s}\left|\nabla w_{L}\right|^{2} d x d y \\
& \leq \beta \int_{\Omega \times\{0\}} \eta^{2}\left(\bar{v}^{+}\right)^{2_{s}^{*}}\left(\bar{v}_{L}^{+}\right)^{2(\beta-1)} d x+\beta \int_{\mathcal{C}} y^{1-2 s}\left|\nabla_{x} \eta\right|^{2}\left(\bar{v}^{+}\right)^{2}\left(\bar{v}_{L}^{+}\right)^{2(\beta-1)} d x d y .
\end{aligned}
$$

By the Poincaré type inequality (see also [6]),

$$
\int_{\mathcal{C}} y^{1-2 s}\left|w_{L}\right|^{2} d x d y \leq \int_{\mathcal{C}} y^{1-2 s}\left|\nabla w_{L}\right|^{2} d x d y .
$$


We derive from (2.15) and (2.16) that

$$
\begin{aligned}
& \int_{B_{R}\left(x_{0}\right) \times(0, \infty)} y^{1-2 s}\left(\bar{v}^{+}\right)^{2}\left(\bar{v}_{L}^{+}\right)^{2(\beta-1)} d x d y \\
& \leq \beta \int_{\Omega \times\{0\}} \eta^{2}\left(\bar{v}^{+}\right)^{2_{s}^{*}}\left(\bar{v}_{L}^{+}\right)^{2(\beta-1)} d x \\
& +\beta r^{-2} \int_{\left(B_{R+r}\left(x_{0}\right) \backslash B_{R}\left(x_{0}\right)\right) \times(0, \infty)} y^{1-2 s}\left(\bar{v}^{+}\right)^{2}\left(\bar{v}_{L}^{+}\right)^{2(\beta-1)} d x d y .
\end{aligned}
$$

Using the filling hole technique, we find that there exist $\theta, \theta_{1}, 0<\theta, \theta_{1}<1$ so that

$$
\begin{aligned}
& \int_{B_{R}\left(x_{0}\right) \times(0, \infty)} y^{1-2 s}\left(\bar{v}^{+}\right)^{2}\left(\bar{v}_{L}^{+}\right)^{2(\beta-1)} d x d y \\
& \leq \beta \theta_{1} \int_{\Omega \times\{0\}} \eta^{2}\left(\bar{v}^{+}\right)^{2_{s}^{*}}\left(\bar{v}_{L}^{+}\right)^{2(\beta-1)} d x \\
& +\theta_{1} \int_{B_{R+r}\left(x_{0}\right) \times(0, \infty)} y^{1-2 s}\left(\bar{v}^{+}\right)^{2}\left(\bar{v}_{L}^{+}\right)^{2(\beta-1)} d x d y .
\end{aligned}
$$

Let

$$
J_{i}=\int_{B_{R+2^{-i_{r}}}\left(x_{0}\right) \times(0, \infty)} y^{1-2 s}\left(\bar{v}^{+}\right)^{2}\left(\bar{v}_{L}^{+}\right)^{2(\beta-1)} d x d y .
$$

Iterating by (2.18), we obtain

$$
\int_{B_{R}\left(x_{0}\right) \times(0, \infty)} y^{1-2 s}\left(\bar{v}^{+}\right)^{2}\left(\bar{v}_{L}^{+}\right)^{2(\beta-1)} d x d y \leq C \beta \int_{\Omega \times\{0\}} \eta^{2}\left(\bar{v}^{+}\right)^{2_{s}^{*}}\left(\bar{v}_{L}^{+}\right)^{2(\beta-1)} d x
$$

provided that $J_{0}$ is finite. Since $R>0$ can be chosen such that $0<R<R_{0}$ for some $R_{0}>0$, we may have from (2.17) and (2.19) that

$$
\begin{aligned}
& \int_{B_{R}\left(x_{0}\right) \times(0, \infty)} y^{1-2 s}\left(\bar{v}^{+}\right)^{2}\left(\bar{v}_{L}^{+}\right)^{2(\beta-1)} d x d y \\
& \leq C \beta^{2}\left(1+r^{-2}\right) \int_{\Omega \times\{0\}} \eta^{2}\left(\bar{v}^{+}\right)^{2_{s}^{*}}\left(\bar{v}_{L}^{+}\right)^{2(\beta-1)} d x .
\end{aligned}
$$

Now, we claim that $\bar{u}^{+} \in L^{\frac{\left(2_{s}^{*}\right)^{2}}{2}}(\Omega)$. Indeed, choosing $\beta=\frac{2_{s}^{*}}{2}$, we have

$$
\begin{aligned}
& \left(\int_{\Omega} \eta^{2}\left(\bar{u}^{+}\left(\bar{u}_{L}^{+}\right)^{\frac{2_{s}^{*}-2}{2}}\right)^{2_{s}^{*}} d x\right)^{\frac{2}{2_{s}^{*}}} \\
& \leq\left(\int_{\Omega} \eta^{2}\left(\bar{u}^{+}\left(\bar{u}_{L}^{+}\right)^{\frac{2_{s}^{*}-2}{2}}\right)^{2_{s}^{*}} d x\right)^{\frac{2}{2_{s}^{*}}}\left(\int_{\Omega} \eta^{2}\left(\bar{u}^{+}\right)^{2_{s}^{*}} d x\right)^{\frac{2_{s}^{*}-2}{2_{s}^{*}}} .
\end{aligned}
$$

Choosing $R=R_{0}>0$ small enough, we have

$$
\int_{B_{2 R}\left(x_{0}\right)}\left(\bar{u}^{+}\right)^{2_{s}^{*}} d x<\frac{1}{2} .
$$

By (2.21) and letting $L \rightarrow+\infty$, we get

$$
\left.\left(\int_{B_{R}\left(x_{0}\right)}\left(\bar{u}^{+}\right)^{\frac{\left(2_{s}^{*}\right)^{2}}{2}}\right) d x\right)^{\frac{2}{2_{s}^{*}}} \leq C \int_{\Omega}\left(\bar{u}^{+}\right)^{2_{s}^{*}} d x<+\infty .
$$


Now let $t=\frac{\left(2_{s}^{*}\right)^{2}}{2\left(2_{s}^{*}-2\right)}, B_{i}=B_{R+2^{-i} r}\left(x_{0}\right)$, and

$$
I_{i}=\left(\int_{B_{i}}\left(\bar{u}^{+}\right)^{\frac{p t \tau^{i}}{t-1}} d x\right)^{\frac{1}{\tau^{i}}}
$$

Iterating $I_{i}$ as before, we see that $\bar{u}^{+} \in \mathbb{L}^{\infty}\left(B_{R}\left(x_{0}\right)\right)$ for $0<R<R_{0}$. Hence, $u^{+} \in \mathbb{L}^{\infty}\left(B_{R}\left(x_{0}\right)\right)$ for $0<R<R_{0}$.

The proof of Theorem 1.2 is complete.

\section{REFERENCES}

[1] H. Brézis and T. Kato, Remarks on the Schrödinger operator with singular complex potentials, J. Math. Pures Appl. 58 (1979), 137-151. MR539217 (80i:35135)

[2] X. Cabré and A. Capella, Regularity of radial minimizers and extremal solutions of semilinear elliptic equations, J. Funct. Anal. 238 (2006), no. 2, 709-733. MR2253739 (2007d:35078)

[3] X. Cabré and Y. Sire, Nonlinear equations for fractional Laplacians I: Regularity, maximum principles, and Hamiltonian estimates. Preprint arXiv:1012.0867, 2010.

[4] X. Cabré and J. Tan, Positive solutions of nonlinear problems involving the square root of the Laplacian, Adv. in Math. 224 (2010), no. 5, 2052-2093. MR2646117 (2011c:35106)

[5] L. Caffarelli and L. Silvestre, An extension problem related to the fractional Laplacian, Comm. Partial Differential Equations 32 (2007), no. 7-9, 1245-1260. MR2354493 (2009k:35096)

[6] A. Capella, J. Dávila, L. Dupaigne and Y. Sire, Regularity of radial extremal solutions for some non-local semilinear equations, Comm. Partial Differential Equations 36 (2011), no. 8, 1353-1384. MR2825595

[7] E. B. Fabes, C. E. Kenig and R. P. Serapioni, The local regularity of solutions of degenerate elliptic equations, Comm. Partial Differential Equations 7 (1982), no. 1, 77-116. MR643158 (84i:35070)

[8] D. Gilbarg and N. S. Trudinger, Elliptic Partial Differential Equations of Second Order, Springer-Verlag, Berlin, 2001. MR.1814364 (2001k:35004)

[9] L. Silvestre, Regularity of the obstacle problem for a fractional power of the Laplace operator, Comm. Pure Appl. Math. 60 (2007), 67-112. MR2270163(2008a:35041)

[10] Xiping Zhu and Jianfu Yang, Regularity for quasilinear elliptic equations involving critical Sobolev exponents, J. Sys. Sci. ES Math. Sci. 9 (1989), no. 1, 47-52. MR994753 (90e:35027)

Department of Mathematics, Jiangxi Normal University, Nanchang, Jiangxi 330022, People's Republic of ChinA

E-mail address: xiaaliang@sina.com

Department of Mathematics, Jiangxi Normal University, Nanchang, Jiangxi 330022 , People's Republic of China

E-mail address: jfyang_2000@yahoo.com 